\title{
Identification of the potential molecular targets for human intervertebral disc degeneration based on bioinformatic methods
}

\author{
JIAXUAN HE ${ }^{1}$, RONGLIANG XUE ${ }^{1}$, SIYUAN LI ${ }^{1}$, JIANRUI LV ${ }^{1}$, YONG ZHANG ${ }^{1}$, \\ LIYING FAN $^{2}$, YUNPENG TENG ${ }^{1}$ and HAIDONG WEI ${ }^{1}$ \\ Departments of ${ }^{1}$ Anesthesiology and ${ }^{2}$ Orthopedics, The Second Affiliated Hospital of Xi'an, \\ Jiaotong University, Xi'an, Shaanxi 710004, P.R. China
}

Received June 16, 2015; Accepted September 30, 2015

DOI: $10.3892 /$ ijmm.2015.2389

\begin{abstract}
The present study aimed to explore potential molecular targets and gain further insights into the mechanism of intervertebral disc degeneration (IDD) progression. Microarray datasets of GSE19943, GSE15227 and GSE34095 were downloaded from the Gene Expression Omnibus database. Differentially expressed genes (DEGs) in 3 IDD specimens compared with 3 controls in GSE34095, DEGs in 7 grade III and 3 grade IV samples compared with 5 grade II samples in GSE19943, and differentially expressed miRNAs in 3 degenerated samples compared with 3 controls in GSE15227 were screened. Grade III- and IV-specific networks were constructed and grade-specific genes were extracted. The network features were analyzed, followed by Gene Ontology (GO) enrichment analysis and pathway enrichment analysis of grade-specific genes and DEGs identified in GSE34095. Furthermore, miRNA-pathway interactions were analyzed using Fisher's exact test. Tumor protein p53 (TP53) was a hub gene in the grade III-specific network and ubiquitin $\mathrm{C}(U B C)$ was identified to be a hub gene in the grade IV-specific network. Six significant features were identified by grade-specific network topology analysis. Grade-specific genes and DEGs were involved in different GO terms and pathways. Differentially expressed miRNAs were identified to participate in 35 pathways, among which 6 pathways were significantly enriched by DEGs, including apoptosis. The present study identified that key genes (TP53 and UBC) and miR-129-5p may participate in the mechanism of IDD progression. Thus, they may be potential therapeutic targets for IDD.
\end{abstract}

Correspondence to: Dr Rongliang Xue, Department of Anesthesiology, The Second Affiliated Hospital of Xi'an, Jiaotong University, 157 West 5th Road, Xi'an, Shaanxi 710004, P.R. China E-mail: rongliangx@163.com

Key words: intervertebral disc degeneration, differentially expressed genes, differentially expressed miRNAs, grade-specific genes, pathway enrichment analysis

\section{Introduction}

Intervertebral disc degeneration (IDD) is characterized by damage of the disc structure, progressive loss of water content and proteoglycan in the extracellular matrix (ECM) (1). IDD is considered a predominant source of spine-related diseases and chronic lower back pain, which causes a major economic and social burden affecting millions of people worldwide (2). Current treatment options for IDD, including discectomy (3), intradiscal electrothermal therapy (4) and arthroplasty (5), only address the clinical symptoms of IDD and remain limited with unpredictable outcomes (6). Therefore, understanding the pathophysiology and molecular mechanism underlying IDD appears to be imperative for diagnosis and developing novel therapeutic approaches.

The intervertebral disc consists of three morphologically distinct regions, which are the nucleus pulposus (NP), annulus fibrosis and cartilaginous endplates (7). The central NP is a gelatinous matrix that is composed of large aggregating proteoglycans and a loose network of collagen. The peripheral annulus fibrosis encases the nucleus pulposus and is rich in type I collagen. The cartilaginous endplates contain the peripheral vasculature, which can nourish the disc (1). The main morphological manifestations of IDD are vertebral instability, disc herniation and spinal stenosis (8). Significant changes in morphology, structure and composition are accompanied by specific changes in the disc with aging and degeneration, including alteration of the elastic modulus and swelling pressure of the nucleus pulposus (9). Recently, histological grading schemes for assessing human IDD have been developed $(10,11)$. A 5-level grading system for lumbar disc degeneration, proposed by Pfirrmann et al (11), was developed according to $\mathrm{T} 2$-weighted magnetic resonance images. Additionally, the Thompson scoring system scores disc degeneration over the spectrum from healthier discs (grades I and II) to advanced degeneration (grade V, the most advanced stage of degeneration) (10). However, there is a lack of studies on the associations of the grades of disc degeneration.

IDD is a complex multi-factorial process (12). Several factors, such as biological, mechanical and genetic factors, are widely considered as significant contributors to the disc degenerative process (1). For example, Bachmeier et al (13) identified that matrix metalloproteinase-3 (MMP-3), which was essential for matrix degeneration, had an essential role in lumbar disc 
herniation and degeneration. Additionally, Takahashi et al (14) revealed that the polymorphism $5 \mathrm{~A}$ allele, which often occurs in the promoter region of the gene that regulates MMP-3 expression, was a possible risk factor for accelerated IDD in the elderly. In addition, Pratsinis et al (15) reported that platelet-derived growth factor, insulin-like growth factor-I and basic fibroblast growth factor could stimulate the proliferation of intervertebral disc cells via the activation of the extracellular-signal regulated kinase and Akt signaling pathways. By contrast, microRNAs (miRNAs) that can regulate RNA degradation or repression of translation have been identified as key regulators in numerous biological processes (16). Recently, Yu et al (17) demonstrated that miR-10b could promote nucleus pulposus cell proliferation by targeting homeobox D10 through the ras homolog family member C-Akt pathway in IDD. However, the molecular mechanism of IDD is not fully understood and further investigations are required.

Recently, Chen et al (18) reported that mitogen-activated protein (MAP) kinase kinase 6 (MAP2K6) and Ras homologous-related BTB domain containing 2 may have significant roles in the progression of grade III and IV disc degeneration, respectively. Additionally, Tang et al (8) demonstrated that differentially expressed genes (DEGs) identified in degenerative disc tissue samples were mainly associated with transforming growth factor- $\beta$ and the ECM. Furthermore, Wang et al (19) reported that deregulated miR-155 could promote Fas-mediated apoptosis in human IDD by targeting Fas-associated death domain-containing protein (FADD) and caspase-3. In the present study, 3 microarray datasets were integrated and microarray analysis was used to identify the DEGs, grade-specific genes and differentially expressed miRNAs in the IDD samples compared with their corresponding controls. In addition, comprehensive bioinformatics was used to analyze the significant functions and pathways, and to analyze the miRNA-target gene-pathway interaction associations. The study aimed to identify the significant genes and miRNA alternations in the progression of IDD and gain more insights into the molecular mechanisms of disc degeneration. Understanding these mechanisms can aid in exploring an appropriate molecular target and developing new therapeutic methods for IDD.

\section{Materials and methods}

Microarray data. Microarray datasets of GSE19943 (19), GSE15227 (20) and GSE34095 (21) were downloaded from the National Center of Biotechnology Information (NCBI), Gene Expression Omnibus (GEO) database (http://www.ncbi. nlm.nih.gov/geo/). The miRNA expression profile GSE19943 included 3 degenerative NP samples that were collected from patients with IDD and 3 NP controls derived from patients with scoliosis, which was based on the platform GPL9946 Exiqon human miRCURY LNA ${ }^{\mathrm{TM}}$ microRNA Array V11.0. The GSE15227 dataset included 5 grade II, 7 grade III, and 3 grade IV discs from patients with herniated discs and IDD that were scored using the Thompson scoring system, which was based on the platform GPL1352 [U133_X3P] Affymetrix Human X3P array. The gene expression profile GSE34095 included 3 degenerative NP samples that were harvested from elderly patients with IDD and 3 non-degenerative samples derived from younger patients with adolescent idiopathic scoliosis as controls, which was based on the platform GPL96 [HG-U133A] Affymetrix Human Genome U133A array.

DEGs and differentially expressed miRNAs screening. The t-test in the limma package (22) was used to identify DEGs in degenerative samples compared with controls in GSE34095, DEGs in 7 grade III and 3 grade IV samples compared with 5 grade II samples in GSE15227, and to identify differentially expressed miRNAs in 3 degenerated samples compared with controls in GSE19943. The false discovery rate (FDR) was applied to perform multiple testing corrections using the Benjamini and Hochberg method (23). The threshold for the DEGs and the differentially expressed miRNAs were set as FDR $<0.05$.

miRNA-target gene and KEGG pathway data collection. Human miRNAs and their associated targets were downloaded from 3 high-quality online miRNA reference databases, which store manual collections of experimentally supported miRNA targets, miRecords (24), miRTarBase (25) and TarBase 6.0 (26). In addition, all pathways and associated genes were downloaded from the Kyoto Encyclopedia of Genes and Genomes (KEGG) database (27).

Grade-specific network construction. DEGs identified in grade III and IV samples were imported into Cytoscape software (28) to create protein-protein interaction (PPI) network visualizations, respectively. The source of the interaction network database was the Search Tool of the Retrieval of Interacting Genes 9.0 database, which is a comprehensive database containing functional links between proteins that are experimentally derived, as well as links predicted by text mining and comparative genomics (29). The threshold was set as confidence score, 0.4. Furthermore, the specific genes that were only enriched in one of the grade-specific networks and the common genes of the 2 grade-specific networks were extracted. The parameters describing the network topology were calculated using the Cytoscape plugin Network Analyzer (30). Logistic regression analysis (31) was conducted by SPSS 19.0 (IBM, Corp., Armonk, NY, USA) to select the most noteworthy network features.

Cluster analysis. To test the availability of these 2 grade-specific networks to reveal different stages of IDD progression, clustering analysis was performed on all genes in the networks and the top $20 \%$ nodes that had a higher degree in the network using Cytoscape plugin Network Analyzer (30), respectively. The results are represented by heat-maps.

GO and pathway enrichment analysis of DEGs and the specific genes. GO and KEGG enrichment analysis were performed for the specific genes using the Database for Annotation, Visualization and Integrated Discovery (DAVID) online tool (32). The threshold was set as $\mathrm{P}<0.05$.

Furthermore, KEGG enrichment analyses were also performed for DEGs that were identified based on GSE34095 using DAVID. The threshold was set as $\mathrm{P}<0.05$.

Measurement of miRNA-pathway interactions. With the identified differentially expressed miRNAs, as well as their target genes and the genes enriched in the KEGG pathways, 
Fisher's exact test (33) was used to assess the corresponding significance of the miRNA pathway.

Fisher's exact test was based on the hypergeometric distribution to combine the results of the proportion of the miRNA target gene in the functional gene set and the proportion of miRNA target gene in the whole genome. A P-value for the null hypothesis of the Fisher's exact test was examined as the genes that belong to the target genes of miRNAs and the genes that belong to the functional genes enriched.

The resulting $\mathrm{P}$-value depicts the probability that the examined pathway is significantly enriched with gene targets of the selected miRNAs, the probability that at least $x$ functional genes are enriched in the $K$ target genes of miRNA. The P-value can be expressed as:

$$
p=1-\sum_{i=0}^{x-1} \frac{\left(\begin{array}{l}
M \\
i
\end{array}\right)\left(\begin{array}{l}
N-M \\
K-i
\end{array}\right)}{\left(\begin{array}{l}
N \\
K
\end{array}\right)}
$$

Where $N$ is the total number of genes, $M$ is the number of genes in the functional genes set and $K$ is the number of target genes of miRNA.

\section{Results}

Screening of DEGs and differentially expressed miRNAs. For GSE15227, 846 and 1,137 DEGs were identified in grade III and IV discs, respectively. For GSE34095, a total of 961 DEGs were identified in the IDD samples compared with the controls. Furthermore, for GSE19943, 77 differentially expressed miRNAs were identified in the degenerative NP samples compared with the controls.

Grade-specific network construction. The grade-specific networks are shown in Figs. 1 and 2. In the present study, 746 grade III-specific genes and 964 grade IV-specific genes were identified. Tumor protein p53 (TP53) was a hub gene in the grade III-specific network and ubiquitin $\mathrm{C}(U B C)$ was identified to be a hub gene in the grade IV-specific network. Additionally, there were 78 common genes in these 2 grade-specific networks. Network topology analysis showed that a total of 16 network features were identified. In addition, statistical regression analysis revealed that 6 significant network features were obtained, as shown in Table I, including average shortest path length, betweenness centrality, closeness centrality, neighborhood connectivity, radiality and stress.

Clustering analysis. The clustering analysis was used to group the genes and samples on the basis of similarities of gene expression. The results of clustering analysis using all the specific genes in these 2 networks are shown in Fig. 3A. The result showed that 2 grade III samples (GSM380301 and GSM380305) were grouped into the region of grade IV samples; however, the correlation was lower compared to the correlation of the grade IV samples. By contrast, the results of clustering analysis using the top $20 \%$ genes with a higher degree in these 2 networks are shown in Fig. 3B. Consistently, the 2 grade III samples, GSM380301 and GSM380305, were grouped into the region of grade IV samples.
Table I. Logistic regression.

\begin{tabular}{lcc}
\hline Feature & P-value & Exp (B) \\
\hline Average shortest path length & $<0.0001$ & 0.050 \\
Betweenness centrality & $<0.0001$ & $<0.0001$ \\
Closeness centrality & $<0.0001$ & $<0.0001$ \\
Neighborhood connectivity & 0.030 & 1.015 \\
Radiality & 0.036 & $<0.0001$ \\
Stress & $<0.0001$ & 1.010 \\
Constant & 0.001 & $1.08 \times 10^{11}$
\end{tabular}

Exp (B) represents odd ratio in the logistic regression model.

Table II. GO terms of grade III-specific genes.

GO term P-value

Angiogenesis

0.007

Adult walking behavior

Retina development in camera-type eye

0.008

Activation of protein kinase activity

0.008

Response to DNA damage stimulus

0.008

Negative regulation of biosynthetic process

0.009

Negative regulation of cellular biosynthetic process

0.011

Negative regulation of macromolecule biosynthetic $\quad 0.014$

process

Positive regulation of protein kinase activity $\quad 0.015$

Positive regulation of kinase activity $\quad 0.020$

Cytokine secretion

0.024

Negative regulation of gene expression $\quad 0.024$

Negative regulation of transcription $\quad 0.025$

Positive regulation of transferase activity $\quad 0.026$

Negative regulation of nucleobase, nucleoside, $\quad 0.029$

nucleotide and nucleic acid metabolic process

Negative regulation of nitrogen compound

0.034

metabolic process

Vasculature development

0.037

Patterning of blood vessels

0.038

Tube morphogenesis

0.039

Regulation of protein kinase activity $\quad 0.043$

Adult locomotory behavior $\quad 0.044$

Actin filament organization $\quad 0.044$

Cell-substrate junction assembly $\quad 0.048$

Negative regulation of macromolecule metabolic $\quad 0.050$ process

GO, Gene Ontology.

GO and pathway enrichment analysis of specific genes. GO and pathway analysis indicated that grade III- and IV-specific genes were significantly enriched in different GO terms and KEGG pathways. A total of $23 \mathrm{GO}$ terms for grade III-specific genes were enriched, including angiogenesis, adult walking behavior and positive regulation of kinase activity (Table II). 


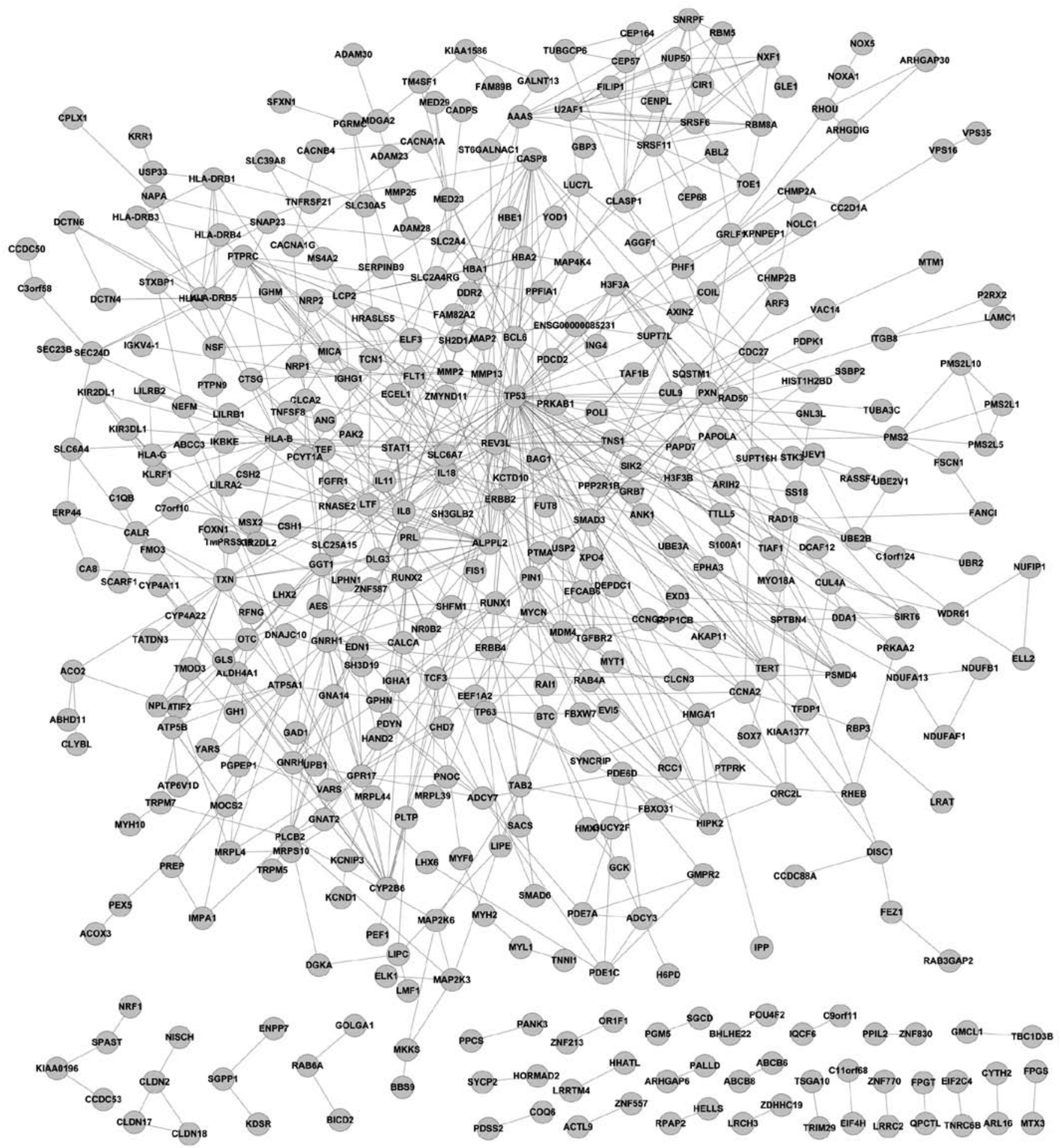

Figure 1. Grade III-specific network.

GO terms for grade IV-specific genes were mainly associated with the regulation of ubiquitin-protein ligase activity, such as positive regulation of ubiquitin-protein ligase activity, and regulation of protein ubiquitination (Table III). In addition, 4 KEGG pathways for grade III-specific genes were significantly enriched, including Alzheimer's disease, oxidative phosphorylation, Huntington's disease and Parkinson's disease. A total of $5 \mathrm{KEGG}$ pathways for grade IV-specific genes were significantly enriched, including viral myocarditis, graft-versus-host disease, type I diabetes mellitus, allograft rejection and cell adhesion molecules (Table IV).

miRNA-pathway-genes complex regulatory associations analysis. Subsequent to combining miRecords, miRTarBase and TarBase 6.0 databases, 5,489 miRNA-target pairs were collected in the study, including 482 miRNAs and 2,331 target genes. miRNA-pathway interactions analysis showed that there were 217 interaction pairs involved in 35 significant 


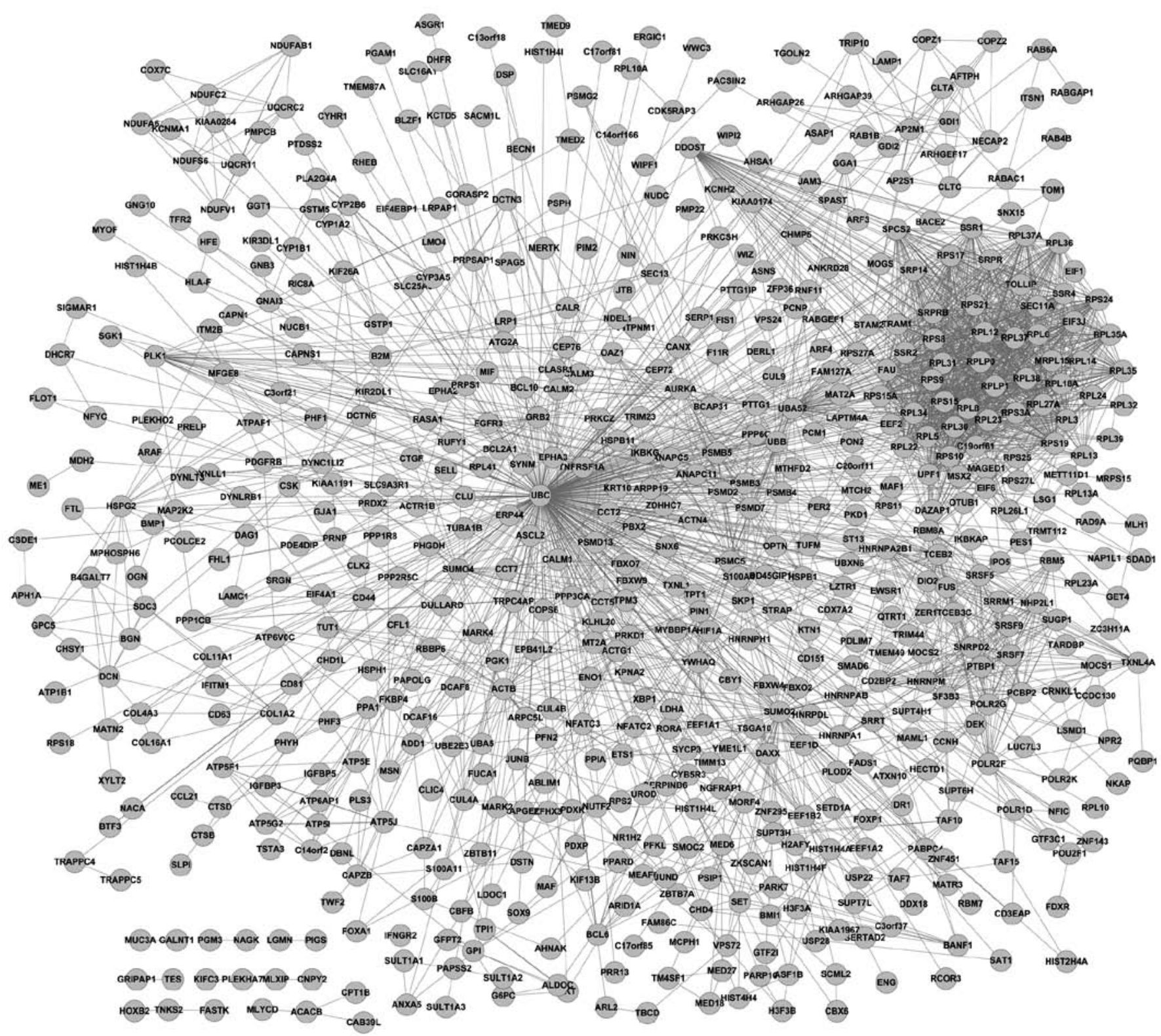

Figure 2. Grade IV-specific network.

pathways. Furthermore, the pathway enrichment analysis for DEGs in the degenerative NP samples compared with the controls showed that 21 KEGG pathways were significantly enriched. There were 6 pathways also regulated by miRNAs, as shown in Table V, including focal adhesion, the ErbB signaling pathway, the calcium signaling pathway, the MAPK signaling pathway, apoptosis and pathways in cancer. Additionally, only miR-129-5p was involved in the calcium signaling pathway, apoptosis and pathways in cancer.

\section{Discussion}

In the present study, microarray analysis showed that a total of 846 and 1,137 DEGs were identified in grade III and IV discs, respectively. In addition, 961 DEGs were identified in the IDD samples in GSE34095 and 77 differentially expressed miRNAs were identified in the IDD samples. TP53 was a hub gene in the grade III-specific network and $U B C$ was identified as a hub gene in grade IV-specific network. Six significant features were identified by grade-specific network topology analysis. Differentially expressed miRNAs were identified to participate in 35 pathways, among which 6 pathways were significantly enriched by DEGs, including apoptosis.

The general concepts, such as centrality, communicability and betweenness, quantify the important features in a network (34). Estrada (35) demonstrated that subgraph centrality could be applied to the identification of essential proteins in PPI networks. Additionally, the threshold for inclusion was $\mathrm{P}<0.05$ (significant) in the logistic regression model (36). In line with the previous study, the present study showed that 6 network features (average shortest path length, betweenness centrality, closeness centrality, neighborhood connectivity, radiality and stress) had significant differences 

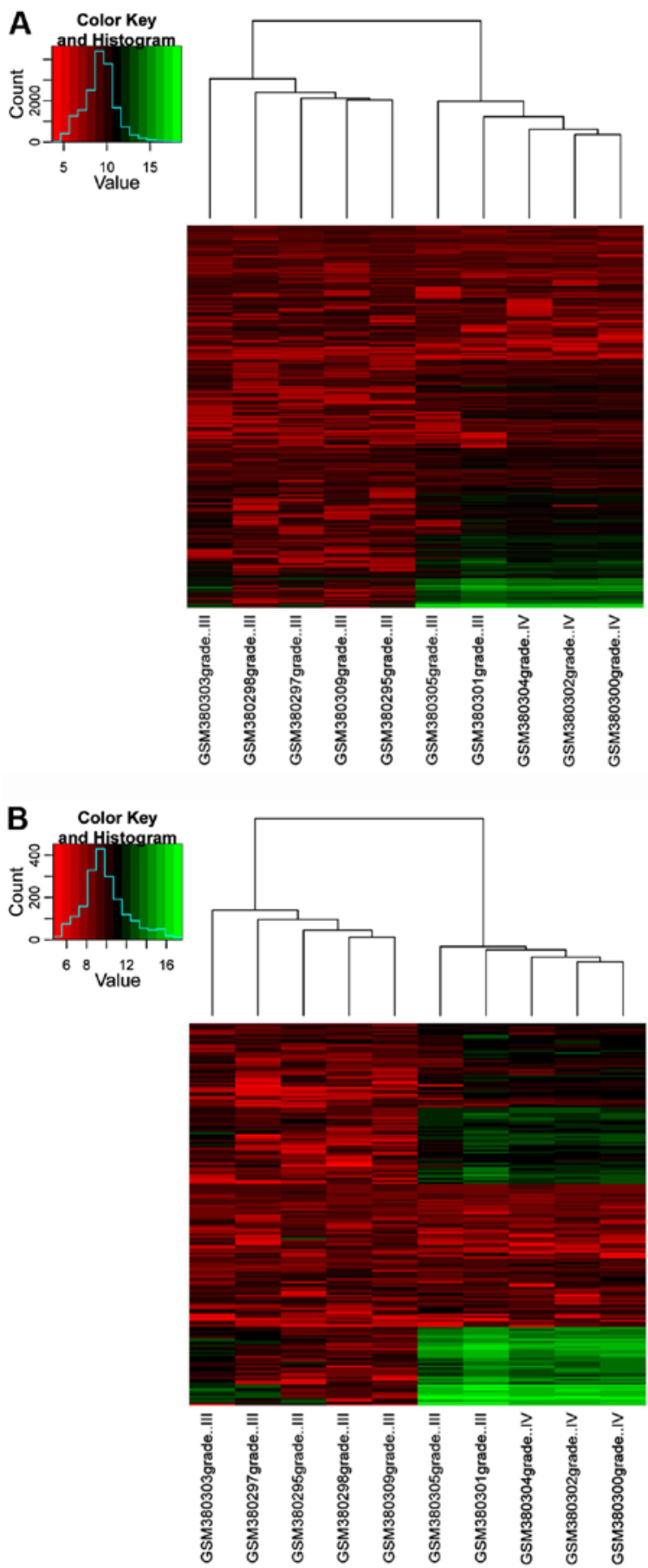

Figure 3. Heat-maps of specific genes. (A) Heat-maps of all the specific genes in 2 grade-specific networks. (B) Heat-maps of the top $20 \%$ specific genes that had higher degrees in the grade-specific networks. The red color indicates a high expression level and the green color indicates a low expression level of a specific gene in a particular specimen.

in grade III- and IV-specific networks (Table I). On this basis, these 6 features may have a significant association with the grade of discs degeneration and could be useful for assessing the IDD grade.

TP53 is a tumor-suppressor protein containing DNA binding, transcriptional activation and oligomerization domains (37). Vaghefi et al (38) revealed that deacetylation of p53 was a nerve growth factor (NGF)-dependent post-translational mechanism of p53 activation. Additionally,
Table III. GO terms of grade IV-specific genes.

\begin{tabular}{lc}
\hline GO terms & P-value \\
\hline Cellular macromolecule localization & 0.036 \\
Cellular protein localization & 0.030 \\
Establishment of protein localization & 0.026 \\
Intracellular protein transport & 0.038 \\
Positive regulation of ligase activity & 0.031 \\
Positive regulation of protein ubiquitination & 0.039 \\
Positive regulation of ubiquitin-protein ligase & 0.019 \\
activity & \\
Positive regulation of ubiquitin-protein ligase & 0.013 \\
activity during mitotic cell cycle & \\
Protein transport & 0.036 \\
Regulation of ligase activity & 0.025 \\
Regulation of protein ubiquitination & 0.022 \\
Regulation of ubiquitin-protein ligase activity & 0.015 \\
Regulation of ubiquitin-protein ligase activity & 0.022 \\
during mitotic cell cycle &
\end{tabular}

GO, Gene Ontology.

Table IV. Pathway enrichment of grade III- and IV-specific genes.

\begin{tabular}{ll}
\hline Pathway & P-value \\
\hline $\begin{array}{l}\text { Grade III } \\
\text { hsa05010: Alzheimer's disease }\end{array}$ \\
hsa00190: Oxidative phosphorylation & 0.009 \\
hsa05016: Huntington's disease & 0.011 \\
hsa05012: Parkinson's disease & 0.012 \\
Grade IV & 0.021 \\
hsa05416: Viral myocarditis & \\
hsa05332: Graft-versus-host disease & $9.37 \times 10^{-4}$ \\
hsa04940: Type I diabetes mellitus & 0.012 \\
hsa05330: Allograft rejection & 0.017 \\
hsa04514: Cell adhesion molecules & 0.040 \\
\hline
\end{tabular}

Richardson et al (39) reported that NGF had increased expression in the painful degenerate intervertebral disc. In addition, Liu et al (40) demonstrated that VEGF and p53 were working simultaneously in neovascularization and infiltration, and accelerating rat IDD progression. In line with the previous study, TP53 was identified as a hub gene in the grade III-specific network, which was derived from the microarray data of GSE15227. Furthermore, specimens of GSE15227 are annulus fibrosus cells in cultures from patients with herniated discs and IDD. Thus, we hypothesized that TP53 may participate in neovascularization and infiltration in annulus fibrosus in IDD progression, which could also help the IDD grading system.

Additionally, the present study identified that the GO terms for grade IV-specific genes were mainly associated with the regulation of ubiquitin-protein ligase activity and 
$U B C$ was a hub gene in the grade IV-specific network derived from the data of GSE15227. UBC encodes a poly-ubiquitin precursor that can conjugate with different residues and lead to various effects within a cell (41). Yew (42) demonstrated that G1- and S-phase events in vertebrates, which were essential for cell proliferation, were specifically mediated by a multitude of regulators associated with ubiquitination, of which $U B C$ was required. Furthermore, Gruber et al (43) reported that cells in IDD lost their ability to proliferate and were subject to senescence. Thus, we hypothesized that $U B C$ may have a crucial role in inhibiting cell proliferation of annulus fibrosus in IDD progression and could be used for grading disc degeneration.

Furthermore, the present study showed that miR-129-5p could participate in different pathways, including apoptosis. The study by Kohyama et al (44) reported that induction of the apoptosis of disc cells had an important role in the pathogenesis of disc degeneration. In addition, Li et al (45) revealed that downregulation of miR-129-5p could inhibit cell growth and induce apoptosis in laryngeal squamous cell carcinoma by targeting adenomatous polyposis coli. In the present study, the miRNA-pathway interaction analysis was based on the information of differentially expressed miRNAs derived from GSE19943 and the pathway enrichment analysis for DEGs in degenerative NP samples was derived from GSE34095. Additionally, tissues of GSE19943 and GSE34095 are NP from patients with IDD and scoliosis. Furthermore, 6 overlapping pathways were identified according to the results of miRNA-pathway interactions analysis and pathway enrichment analysis for DEGs, including apoptosis. Therefore, we hypothesized that miR-129-5p may have a crucial role in modulating cell apoptosis in NP in IDD progression and miR-129-5p could be a potential therapeutic target for IDD. However, more experiments and further investigations are required to verify this finding.

In conclusion, the present study identified that key genes (TP53 and UBC) and miR-129-5p may participate in the mechanism of IDD progression. Significant network features identified in the study may aid in assessing the grade of disc degeneration. TP53 may have an essential role in neovascularization and infiltration in annulus fibrosus in grade III of IDD progression and could help the IDD grading system. Additionally, $U B C$ may have a crucial role in inhibiting cell proliferation of annulus fibrosus in IDD progression and could be used to grade disc degeneration. Furthermore, miR-129-5p may have a crucial role in modulating cell apoptosis in NP in IDD progression and miR-129-5p could be a potential therapeutic target for IDD. However, further experiments and studies are required to confirm these results.

\section{Acknowledgements}

The present study was supported by the National Natural Science Foundation of China: The effect and mechanism of cytoskeletal elements in the mechanotransduction pathway within intervertebral disc cells and its role in intervertebral disc degeneration (grant no. 81471131) and the National Natural Science Foundation of China: The effect and mechanism of chondroitin sulphate proteoglycans in stem cell niche during stem cell differentiation (grant no. 31300675).

\section{References}

1. Hadjipavlou AG, Tzermiadianos $\mathrm{MN}$, Bogduk $\mathrm{N}$ and Zindrick MR: The pathophysiology of disc degeneration: A critical review. J Bone Joint Surg Br 90: 1261-1270, 2008.

2. Wang SZ, Rui YF, Tan Q and Wang C: Enhancing intervertebral disc repair and regeneration through biology: Platelet-rich plasma as an alternative strategy. Arthritis Res Ther 15: 220, 2013.

3. Yorimitsu E, Chiba K, Toyama Y and Hirabayashi K: Long-term outcomes of standard discectomy for lumbar disc herniation: A follow-up study of more than 10 years. Spine 26: 652-657, 2001.

4. Karasek M and Bogduk N: Twelve-month follow-up of a controlled trial of intradiscal thermal anuloplasty for back pain due to internal disc disruption. Spine 25: 2601-2607, 2000.

5. Anderson PA and Rouleau JP: Intervertebral disc arthroplasty. Spine 29: 2779-2786, 2004.

6. Shimer AL, Chadderdon RC, Gilbertson LG and Kang JD: Gene therapy approaches for intervertebral disc degeneration. Spine 29: 2770-2778, 2004.

7. Choi YS: Pathophysiology of degenerative disc disease. Asian Spine J 3: 39-44, 2009.

8. Tang Y, Wang S, Liu Y and Wang X: Microarray analysis of genes and gene functions in disc degeneration. Exp Ther Med 7: 343-348, 2014

9. Antoniou J, Epure LM, Michalek AJ, Grant MP, Iatridis JC and Mwale F: Analysis of quantitative magnetic resonance imaging and biomechanical parameters on human discs with different grades of degeneration. J Magn Reson Imaging 38: 1402-1414, 2013.

10. Thompson JP, Pearce RH, Schechter MT, Adams ME, Tsang IK and Bishop PB: Preliminary evaluation of a scheme for grading the gross morphology of the human intervertebral disc. Spine 15: 411-415, 1990.

11. Pfirrmann CW, Metzdorf A, Zanetti M, Hodler J and Boos N: Magnetic resonance classification of lumbar intervertebral disc degeneration. Spine 26: 1873-1878, 2001.

12. Adams MA and Roughley PJ: What is intervertebral disc degeneration, and what causes it? Spine 31: 2151-2161, 2006.

13. Bachmeier BE, Nerlich A, Mittermaier N, Weiler C, Lumenta C, Wuertz K and Boos N: Matrix metalloproteinase expression levels suggest distinct enzyme roles during lumbar disc herniation and degeneration. Eur Spine J 18: 1573-1586, 2009.

14. Takahashi M, Haro H, Wakabayashi Y, Kawa-uchi T, Komori H and Shinomiya K: The association of degeneration of the intervertebral disc with $5 \mathrm{a} / 6 \mathrm{a}$ polymorphism in the promoter of the human matrix metalloproteinase-3 gene. J Bone Joint Surg Br 83: 491-495, 2001.

15. Pratsinis $\mathrm{H}$ and Kletsas D: PDGF, bFGF and IGF-I stimulate the proliferation of intervertebral disc cells in vitro via the activation of the ERK and Akt signaling pathways. Eur Spine J 16: 1858-1866, 2007.

16. van Rooij E and Kauppinen S: Development of microRNA therapeutics is coming of age. EMBO Mol Med 6: 851-864, 2014.

17. Yu X, Li Z, Shen J, Wu WK, Liang J, Weng X and Qiu G: MicroRNA-10b promotes nucleus pulposus cell proliferation through RhoC-Akt pathway by targeting HOXD10 in intervetebral disc degeneration. PLoS One 8: e83080, 2013.

18. Chen Y, Chen K, Li M, Li C, Ma H, Bai YS, Zhu XD and Fu Q: Genes associated with disc degeneration identified using microarray gene expression profiling and bioinformatics analysis. Genet Mol Res 12: 1431-1439, 2013.

19. Wang HQ, Yu XD, Liu ZH, Cheng X, Samartzis D, Jia LT, Wu SX, Huang J, Chen J and Luo ZJ: Deregulated miR-155 promotes Fas-mediated apoptosis in human intervertebral disc degeneration by targeting FADD and caspase-3. J Pathol 225: 232-242, 2011.

20. Gruber HE, Hoelscher G, Loeffler B, Chow Y, Ingram JA, Halligan W and Hanley EN Jr: Prostaglandin E1 and misoprostol increase epidermal growth factor production in $3 \mathrm{D}$-cultured human annulus cells. Spine J 9: 760-766, 2009.

21. Tsai TT, Lai PL, Liao JC, Fu TS, Niu CC, Chen LH, Lee MS, Chen WJ, Fang HC, Ho NY, et al: Increased periostin gene expression in degenerative intervertebral disc cells. Spine J 13: 289-298, 2013

22. Gentleman R, Carey VJ, Huber W, Irizarry RA and Dudoit S (eds): Bioinformatics and computational biology solutions using $\mathrm{R}$ and Bioconductor. Springer, 2005.

23. Benjamini Y and Hochberg Y: Controlling the false discovery rate: A practical and powerful approach to multiple testing. J R Stat Soc B 57: 289-300, 1995 
24. Xiao F, Zuo Z, Cai G, Kang S, Gao X and Li T: miRecords: An integrated resource for microRNA-target interactions. Nucleic Acids Res 37: D105-D110, 2009.

25. Hsu SD, Lin FM, Wu WY, Liang C, Huang WC, Chan WL, Tsai WT, Chen GZ, Lee CJ, Chiu CM, et al: miRTarBase: A database curates experimentally validated microRNA-target interactions. Nucleic Acids Res 39: D163-D169, 2011.

26. Vergoulis T, Vlachos IS, Alexiou P, Georgakilas G Maragkakis M, Reczko M, Gerangelos S, Koziris N, Dalamagas T and Hatzigeorgiou AG: TarBase 6.0: Capturing the exponential growth of miRNA targets with experimental support. Nucleic Acids Res 40: D222-D229, 2012.

27. Kanehisa M and Goto S: KEGG: Kyoto encyclopedia of genes and genomes. Nucleic Acids Res 28: 27-30, 2000.

28. Smoot ME, Ono K, Ruscheinski J, Wang PL and Ideker T: Cytoscape 2.8: New features for data integration and network visualization. Bioinformatics 27: 431-432, 2011.

29. Franceschini A, Szklarczyk D, Frankild S, Kuhn M, Simonovic M, Roth A, Lin J, Minguez P, Bork P, von Mering C, et al: STRING v9.1: Protein-protein interaction networks, with increased coverage and integration. Nucleic Acids Res 41: D808-D815, 2013.

30. Assenov Y, Ramírez F, Schelhorn S-E, Lengauer T and Albrecht M: Computing topological parameters of biological networks. Bioinformatics 24: 282-284, 2008

31. Huang Y, Pepe MS and Feng Z: Logistic regression analysis with standardized markers. Ann Appl Stat 7: 7, 2013.

32. Sherman BT, Huang da W, Tan Q, Guo Y, Bour S, Liu D, Stephens R, Baseler MW, Lane HC and Lempicki RA: DAVID Knowledgebase: A gene-centered database integrating heterogeneous gene annotation resources to facilitate high-throughput gene functional analysis. BMC Bioinformatics 8: 426, 2007.

33. Blevins L and McDonald C: Fisher's Exact Test: an easy-to-use statistical test for comparing outcomes. MD Comput 2: 15, 1985.

34. Estrada E and Higham DJ: Network properties revealed through matrix functions. SIAM Rev 52: 696-714, 2010.
35. Estrada E: Virtual identification of essential proteins within the protein interaction network of yeast. Proteomics 6: 35-40, 2006.

36. Tailor A, Jurkovic D, Bourne TH, Collins WP and Campbell S: Sonographic prediction of malignancy in adnexal masses using multivariate logistic regression analysis. Ultrasound Obstet Gynecol 10: 41-47, 1997.

37. Dumont P, Leu JI, Della Pietra AC III, George DL and Murphy M: The codon 72 polymorphic variants of p53 have markedly different apoptotic potential. Nat Genet 33: 357-365, 2003.

38. Vaghefi H and Neet KE: Deacetylation of p53 after nerve growth factor treatment in PC12 cells as a post-translational modification mechanism of neurotrophin-induced tumor suppressor activation. Oncogene 23: 8078-8087, 2004.

39. Richardson SM, Doyle P, Minogue BM, Gnanalingham K and Hoyland JA: Increased expression of matrix metalloproteinase-10, nerve growth factor and substance $\mathrm{P}$ in the painful degenerate intervertebral disc. Arthritis Res Ther 11: R126, 2009.

40. Liu XW, Kang J, Fan XD and Sun LF: Expression and significance of VEGF and p53 in rat degenerated intervertebral disc tissues. Asian Pac J Trop Med 6: 404-406, 2013.

41. Marinovic AC, Zheng B, Mitch WE and Price SR: Ubiquitin (UbC) expression in muscle cells is increased by glucocorticoids through a mechanism involving Sp1 and MEK1. J Biol Chem 277: 16673-16681, 2002.

42. Yew PR: Ubiquitin-mediated proteolysis of vertebrate G1- and S-phase regulators. J Cell Physiol 187: 1-10, 2001.

43. Gruber HE, Ingram JA, Norton HJ and Hanley EN Jr: Senescence in cells of the aging and degenerating intervertebral disc: Immunolocalization of senescence-associated $\beta$-galactosidase in human and sand rat discs. Spine 32: 321-327, 2007.

44. Kohyama K, Saura R, Doita M and Mizuno K: Intervertebral disc cell apoptosis by nitric oxide: Biological understanding of intervertebral disc degeneration. Kobe J Med Sci 46: 283-295, 2000.

45. Li M, Tian L, Wang L, Yao H, Zhang J, Lu J, Sun Y, Gao X, Xiao H and Liu M: Down-regulation of miR-129-5p inhibits growth and induces apoptosis in laryngeal squamous cell carcinoma by targeting APC. PLoS One 8: e77829, 2013. 